(C2010 IEEE. Personal use of this material is permitted. However, permission to reprint/republish this material for advertising or promotional purposes or for creating new collective works for resale or redistribution to servers or lists, or to reuse any copyrighted component of this work in other works must be obtained from the IEEE. 


\section{An Exploratory Investigation of the Organizational Impacts of Collaborative Information Technology Utilization in Australian Organizations}

\author{
Deepinder S. Bajwa \\ Western Washington University \\ deepinder.bajwa@wwu.edu
}

\author{
Graham Pervan \\ Curtin University of Technology \\ g.pervan@curtin.edu.au
}

\author{
L. Floyd Lewis \\ Western Washington University \\ floyd.lewis@wwu.edu
}

\begin{abstract}
In this paper, we extend the existing literature by exploring the organizational-level impacts of Collaborative Information Technology (CIT). We consider CIT as any information technology (IT) that supports collaboration tasks. Data was collected from 73 organizations in Australia to access organizational impacts of CITs. Our results indicate that CIT use for primary collaborative tasks (information and knowledge sharing, decision-making, report writing/information pooling), secondary collaborative tasks (communications, scheduling, and monitoring progress), and tertiary collaborative tasks (issue resolution and discussion and brainstorming) is positively and significantly associated with strategic, efficiency-oriented, and effectiveness-oriented organizational level impacts. Implications of our findings are discussed for practice and research.
\end{abstract}

\section{Introduction}

In this paper we explore the organizational level impacts of Collaborative Information Technologies (CITs) in Australian organizations. Broadly, CITs can be defined as IT applications that support teams/groups engaged in accomplishing tasks/projects. As such, CITs provide a wide range of functionalities including: communication, coordination, decision making, information pooling, amongst others. There are many types of CITs that organizations can deploy, offering a range of functionalities to support teams as they engage in accomplishing tasks in environments with varying degrees of virtuality. Organizations have been using CITs for decades. For example, audio conferencing, video conferencing, proprietary groupware, group support systems etc. have been around for many years. However, most of these traditional CITs have been popular only amongst focused groups of end-users in organizations. Ever since the advent of the Internet, many new tools have emerged, increasing the range and reach of the new generation of CITs to many organizational end-users. There is no scarcity of case evidence that CITs like intranets, extranets, web conferencing, data conferencing, and other online web-based tools, are becoming increasingly accessible to a greater proportion of end-users in modern organizations. While some of these options that may provide specific functionality to support teams and even though integrated e-collaboration tools are beginning to emerge [18], a recent survey of over 200 CIOs and IT decision makers found that lack of integration between the various CIT options available continues to be of concern [27].

The increasing popularity of CITs is evident as many organizations are moving forward to make large investments in these technologies [14]. Despite these trends, their organization level impacts remain largely unexplored. The majority of the research efforts on CIT use have been aimed at understanding the performance of teams with the group(s) being the unit of analysis [6][16][25]. There have been only a few large scale organizational level studies exploring their adoption and use. Moreover, for the most part, these organizational level studies have investigated specific/individual CITs, despite the notion that most collaborative efforts should typically be supported by multiple tools. Some organizational level studies exploring adoption of multiple CITs have been undertaken [3][4] but, to our knowledge, there are no large scale organizational level empirical studies exploring CIT impacts. A few recent studies have focused on CIT impacts on individuals and groups [1][5][24]. Although it is critical to understand group level impacts of CITs, such impacts may not translate to higher order organizational level impacts unless utilization of CITs spreads to a critical mass of endusers. This may occur if these tools become institutionalized amongst a greater proportion of organizational members.

Accordingly, this paper reports on our efforts to explore organizational level impacts of CITs in Australia. The paper is organized as follows. The next 
section briefly reviews the existing literature on CIT impacts. This is followed by a description of the premise of our research. We then outline our study methodology, analysis of data, and discuss our findings. The conclusion section focuses on the implications for research and practice, the limitations of our exploratory study, and identifies directions for future research.

\section{CIT impacts}

Many research studies have explored impacts of CITs use on group performance (for a review see [10][11][21]). In a recent study of 365 group managers in a large multinational corporation, effective use of CITs was found to directly influence groups' process efficiency, situational awareness, and project effectiveness [21]. After a review of the relevant literature, it was argued that CIT functionalities like chat, email, scheduling, conferencing, file sharing, and workspace sharing reduces the cost, time, and effort needed to perform group tasks, thereby greatly enhancing the efficiency of the collaboration process [21]. Similarly, the same set of CIT functionalities helps group members to "stay aware of their surroundings" and "jointly access real-time information about their surroundings" ([21], p3), thereby increasing the situational awareness of group members working together to accomplish tasks. Finally, the same CIT functionalities also tend to allow groups to create, access, and share project knowledge so that they can make better decisions, thereby positively influencing the effectiveness of projects. The impact of CITs on the collaboration process, product, and innovation has also been recognized by others. In general, CITs are believed to positively influence group creativity, which in turn can "facilitate multidisciplinary innovation and reduce barriers and inefficiencies" ([23], p1) when group members collaborate [11].

While substantial evidence exists on group-level impacts of CITs, their organizational level impacts remain largely unexplored. In one of the earlier studies of Lotus Notes (a single CIT - proprietary groupware) in an organization, it was found that implementing CIT can have "efficiency" and "effectiveness" related impacts on the organization [26]. In a more comprehensive review of case studies of Lotus Notes in eighteen organizations, Karsten [17] found that organizational-level impacts were not consistent across all the organizations. Significant changes in work practices, processes, and structure were observed in organizations only when Notes use was "extensive and engaged". Our motivation for the present study is to build on the past research and attempt to understand more about the types of organization level impacts brought about by varying level of use of multiple CITs and the nature of association between the use of CITs and their impacts on organizations.

\section{Research premise}

In general, the impact of IT on organizational performance has been widely researched [7]. While the results have been somewhat mixed, it has been proposed that extensive use of an IT can lead to greater net positive benefits [8] for the adopting individual, unit, or organization. The original DeLone and McLean model of Information System (IS) success has been a popular foundation for many IS studies. While the model has been applied (and to some extent validated and even critiqued) by many researchers, it has endured the test of time and was recently slightly modified after a ten year update [9]. The basic premise underlying the model suggests that IS quality (information, system, and service) influences: intention to use, actual use and user satisfaction with the IS. Use of IS and user satisfaction with the system then lead to IS impacts (or net benefits) at the individual level and at the organizational level. The premises of the model tend to support the findings from an early study of CIT use (specifically use of Lotus Notes) that CIT use does, in fact, result in organization-level impacts [26], but it may not necessarily produce the same outcomes in all organizations [17]. Therefore our objective here is to address the following research questions:

1] What impacts does CIT use have on organizations?

2] What is the nature of the relationship between CIT use and the impacts they bring about in organizations?

\section{Methodology}

The research initiative to explore CIT impacts was initially undertaken in 2006. As our first initiative, we first conducted two case studies in the US to understand more about the broad organization context, the IT infrastructure, CIT use, and organization level impacts of CITs. The organizations varied significantly in the size and scope of their geographical operations. Telephone interviews were conducted with five managers in a large organization and one manager in a small organization. Each participant was sent a detailed document of our research agenda and sample semistructured questions. Average time of each interview was about forty five minutes and all the interviews were recorded. This was followed by two case studies 
in Australia in 2007. A similar interview process was adopted except that the interviews were conducted face-to-face with the senior most IT executive in each of the two organizations. Average time for each interview was about sixty minutes. The conversations were recorded. In all the cases, we found substantial support to address the research questions outlined above.

In order to develop a measuring instrument for our survey, we adopted the approach suggested by Sethi and King [23]. The first step involved a thorough review of the literature to indentify validated measures for our study constructs. Since our constructs had not been explored before in the context of CITs, we developed our own measures. Six researchers participated in developing these measures. All of them have been involved in CIT research for over a decade. Over a period of three months, the research team formulated relevant items from the practitioner and academic literature. The resulting survey instrument was then pilot tested in three Australian organizations. Two of these organizations were the same as our case study organizations. Feedback from the pilot study participants was then used to slightly modify the original instrument. We next focus on the resulting construct measures utilized in this study.

\subsection{Measures}

Eighteen items were formulated to measure CITs impacts based upon prior research on CITs and the relevant literature [2][17][26]. These items focused on: improvements in existing products/services, improved relationships with customers, suppliers, and business partners, improvements in existing business processes, structural changes, time savings, quick reaction to changes, speed of decision-making, increased productivity, appropriate responses to changes, facilitation of innovations, improved quality of decisions, marketing the right products/services, increased fragmentation of work, increased information overload, decreased management control, and decreased independence. While the majority of impact items measured positive impacts, four of them measured negative impacts. A five point Likert-type scale (strongly disagree to strongly agree) was used for all the eighteen items to measure the level of agreement with each of the impacts resulting from the use of IT to support collaboration (i.e. the use of CITs).

An approach paralleling the "function-centric" view [15] was used to measure CIT use. We identified several processes or tasks that underline collaboration. Once again we reviewed the relevant literature to identify collaboration processes/tasks to formulate item measures [10][13][19]. It must be noted here that the existing literature does not conceptually or definitionally differentiate between collaboration tasks and processes and we have not tried to make any such distinction here. In addition to this, we tapped on our own experience on IT enabled collaboration to identify item measures.

Eleven items that comprehensively captured collaboration tasks were identified. These included: communications, information and knowledge sharing, decision-making, report writing/information pooling, planning, scheduling, progress monitoring, time management, issue resolution, discussion and brainstorming, and business process redesign. A five point scale (1=never used, $2,3=$ occasionally used, 4 , $5=$ always used) was deployed to measure the extent to which IT is used to support each of these tasks in collaboration. The majority of the tasks identified in our research are supported by functionalities common to many CITs (see [21]). In addition to the above measures we also captured data on availability and use of various CITs, the implementation of CITs in the organization, and the organizational profile of the responding firms.

\subsection{Data Collection}

An email along with a hard copy of the survey was mailed to CIOs of 500 of the top 1000 organizations in Australia. The email provided a link to the electronic version of our survey in case the respondents preferred completing the survey electronically. A cover letter was included in the mailed surveys. This letter explained the purpose of our study. Clear instructions (including definition of IT supported collaboration, voluntary participation, confidentiality of responses etc.) for the respondents were incorporated in the cover letter and they were requested to forward the survey to the executive most knowledgeable about IT support collaboration in their organization if other than themselves. These instructions were also included on the web survey. A second hard copy mailing was sent out after about a month.

A total of 83 responses (web and traditional mail) were received. Sixty eight questionnaires were returned unopened possibly because the addressed executive could not be located. Ten survey responses were discarded due to substantial missing data. As a result, 73 usable responses were received from four hundred and thirty two surveys that reached the addressed executives. This represents a response rate of about $16.9 \%$. We conducted non-response bias tests and found no significant differences in majority of the study variables between the early and late respondents. 


\section{Results}

In this section, we elaborate on the responses from our study sample. The emphasis is on the profile of our respondents, their organization size, qualitative assessment of IT support for collaborative tasks and organizational impacts of CIT use, and statistical techniques/procedures deployed to test associations between CIT use and organizational impacts of CIT.

\subsection{Response profile}

Although our survey had been mailed to the CIO in all the organizations, our respondents held a wide range of positions, indicating that the survey had actually been forwarded to the executive that (in all probability) was most knowledgeable about IT supported collaborative work. While we received fifteen responses from CIOs, other respondents included senior IT managers, Chief Financial Officers (CFOs), IT directors, Enterprise IT heads, IT strategy managers, amongst others. A wide range of industries were represented in the responses. These included: aviation, manufacturing, financial services, engineering, utilities, education, oil and gas, mining, healthcare, government, legal services, retail, electronics, defense, and leisure and entertainment. Sixty seven respondents reported the number of employees in their respective organizations. Thirteen of the responding organizations had less than 500 employees, forty four of them had employees between 501 and 5000, while ten organizations had employees in excess of 5000. As for the number of IT employees, sixty eight organizations reported the full time in-house IT professionals in their respective organizations. Twenty nine of them had less than 50 in-house $\mathrm{T}$ professionals, fifteen had between 50 and 100 in-house IT professionals, and twenty four had over 100 full time in-house IT staff.

Figure 1 shows the level of use of IT (occasionally used versus always used) to support collaboration in our responding organizations while figure 2 show the agreement and disagreement amongst organizationlevel impacts from the use of IT to support collaboration [or CIT use] in our responding organizations. Overall, our responding organizations had wide variation in reported CIT use and their organization level impacts.

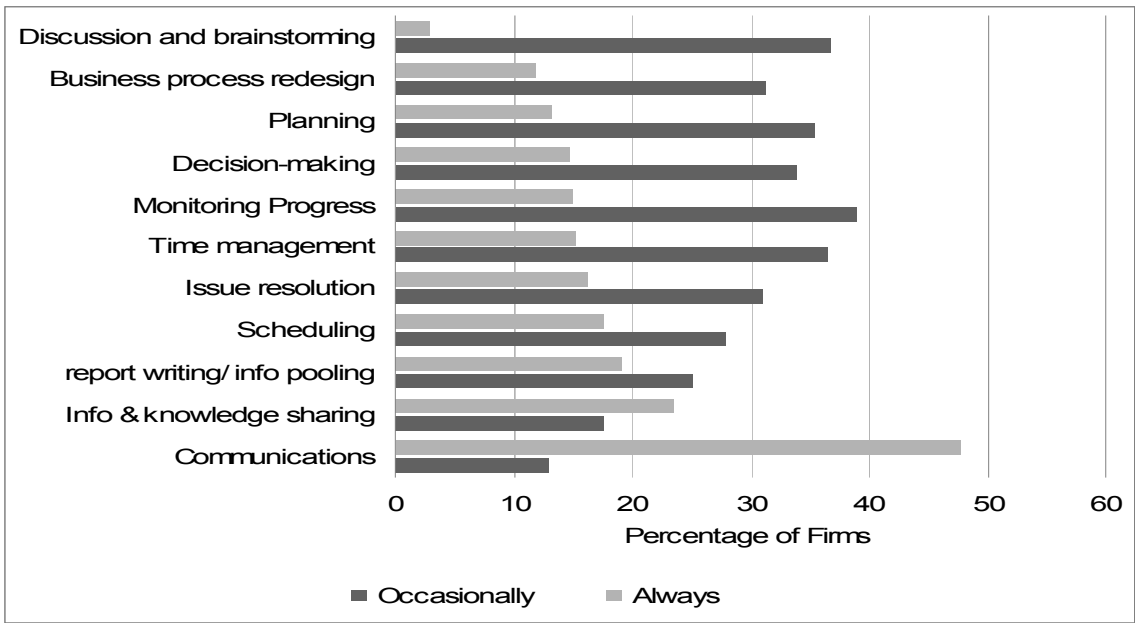

Figure 1. IT use to support collaboration tasks

\subsection{Data Analysis}

The eighteen items used to measure CIT impacts were subjected to principle component factor analysis. In order to justify factor analysis of items measuring CIT impacts, Bartlett test of sphericity was conducted on all the 18 items to detect for the presence of correlations. The Bartlett's test of sphericity resulted in approximate chi-square of 542.89, significant at 0.000 . The Kaiser-Meyer-Olkin measure of sampling adequacy was 0.72 (well above the 0.6 level recommended by Hair et.al [12], suggesting that the items are suitable for factor analysis. To further test for the suitability of factor analysis, the measure of sampling adequacy (MSA) was observed in the antiimage correlation matrix. All the indices were found to be above the minimum 0.50 threshold, once again supporting the use of factor analysis on all the 18 items. Results of the factor analysis (with varimax rotation) are shown in the table 1 along with the coefficient of reliability as measured by Cronbach's alpha. The 18 items loaded on 5 separate factors. 
These were labeled as efficiency-oriented impacts, work-related impacts, and isolation impacts. All the strategic impacts, effectiveness-oriented impacts, loadings were above the minimum level of 0.50 [20].

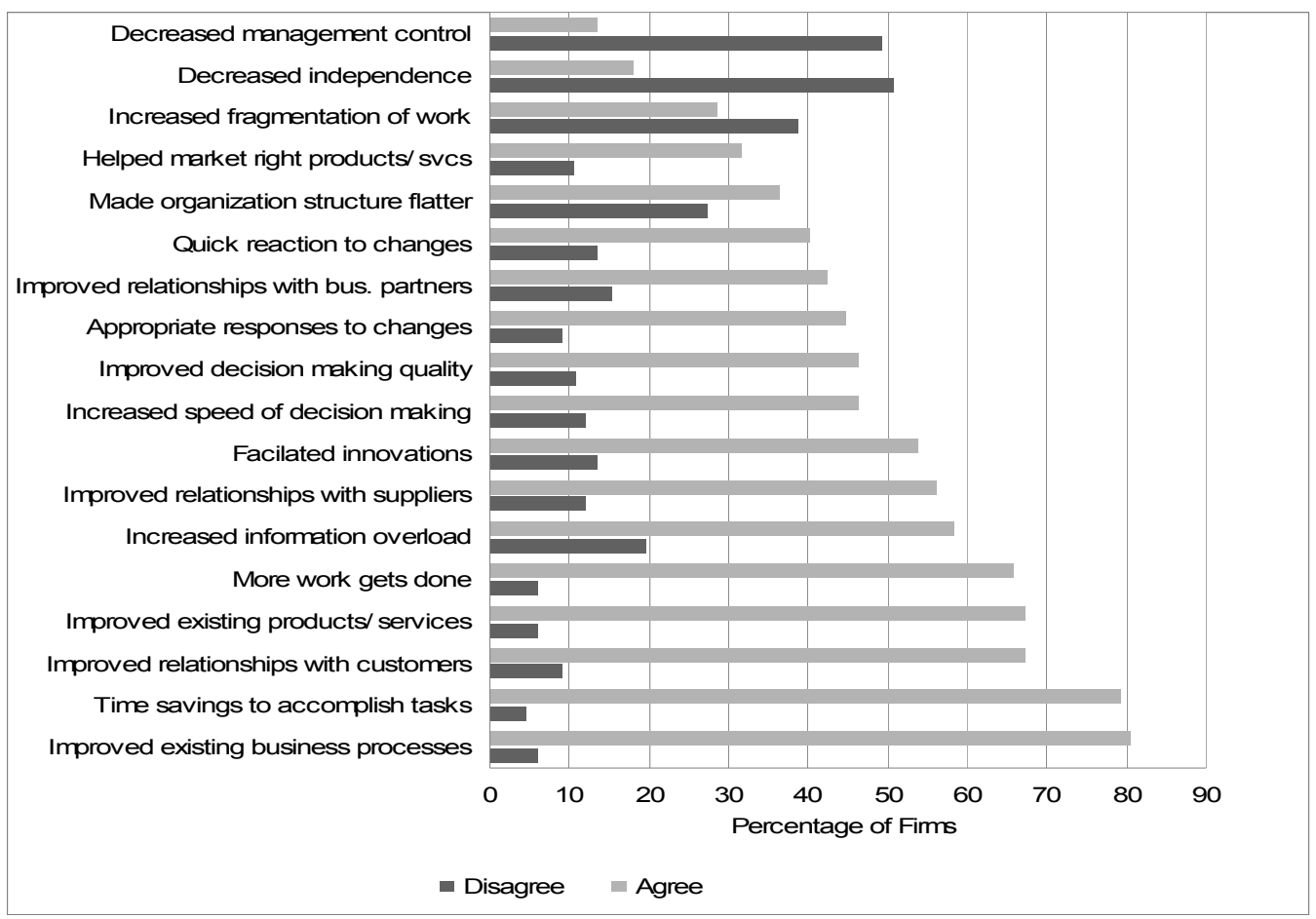

Figure 2. Organization level impacts of IT support collaboration

Table 1. Factor analysis of CIT impacts

\begin{tabular}{|c|c|}
\hline Variables & Loadings \\
\hline \multicolumn{2}{|l|}{ Efficiency-oriented Impacts [Eigen value $=5.81]$, Alpha $=0.87$} \\
\hline - Time saving to accomplish task & 0.62 \\
\hline - $\quad$ Quick reaction to changes & 0.87 \\
\hline - Faster decision-making & 0.85 \\
\hline - More work accomplished & 0.65 \\
\hline - $\quad$ More appropriate changes to responses & 0.75 \\
\hline \multicolumn{2}{|l|}{ Strategic $/$ mpacts [Eigen value $=2.76$ ], Alpha $=0.84$} \\
\hline - Improved existing products/services & 0.69 \\
\hline - Improved relationships with customers & 0.67 \\
\hline - Improved relationships with suppliers & 0.84 \\
\hline - Improved relationships with business partners & 0.83 \\
\hline - Helped market right products/services & 0.56 \\
\hline \multicolumn{2}{|l|}{ Effectiveness-oriented $/$ mpacts[Eigen value $=1.56]$, Alpha $=0.62$} \\
\hline - Improved existing business processes & 0.59 \\
\hline - Flatter organization structure & 0.79 \\
\hline Facilitated innovations & 0.56 \\
\hline \multicolumn{2}{|l|}{ Work-related Impacts [Eigen value $=1.23]$, Alpha } \\
\hline - Increased fragmentation of work & 0.90 \\
\hline Increased information overload & \\
\hline \multicolumn{2}{|l|}{ Isolation Impacts [Eigen value $=1.05]$, Alpha $=0.62$} \\
\hline - Decreased management control & 0.55 \\
\hline - Decreased independence & \\
\hline
\end{tabular}


Similarly, in order to justify factor analysis of items measuring CIT use, Bartlett test of sphericity was conducted on all the eleven items to detect for the presence of correlations. The Bartlett's test of sphericity resulted in approximate chi-square of 302.17 , significant at 0.000 . The Kaiser-Meyer-Olkin measure of sampling adequacy was 0.85 (well above the 0.6 level recommended by Hair et.al [12]), suggesting that the items are suitable for factor analysis. To further test for the suitability of factor analysis on the eleven items, the measure of sampling adequacy (MSA) was observed in the anti-image correlation matrix. All the indices were above 0.81, once again supporting the use of factor analysis on all the eleven items.

Table 2 shows the results of the factor analysis with varimax rotation. Eight of the eleven items loaded on three separate factors. Three items (planning, time management, and business process re-design) loaded equally on more than one factor or had loadings below 0.50 . These three items were excluded from any further analysis. The three factors were labeled as primary tasks, secondary tasks, and tertiary tasks. All the factor loadings were above the 0.50 minimum thresholds for exploratory research.

Table 2. Factor analysis of tasks

\begin{tabular}{|c|l|}
\hline Variables & Loadings \\
\hline Primary Tasks [Eigen value = 4.97], Alpha = 0.78 & \\
$-\quad$ Information and knowledge sharing & 0.83 \\
$-\quad$ Decision-making & 0.76 \\
- Report writing/information pooling & 0.67 \\
Secondary Tasks [Eigen value = 1.34], Alpha = 0.72 & \\
$-\quad$ Communications & 0.62 \\
$-\quad$ Scheduling & 0.88 \\
- Monitoring progress & 0.63 \\
Tertiary Tasks [Eigen value = 1.014], Alpha = 0.68 & \\
$-\quad$ Issue resolution & 0.81 \\
$-\quad$ Discussion and brainstorming & 0.81 \\
\hline
\end{tabular}

Table 3. Descriptive statistics

\begin{tabular}{|l|c|c|c|}
\hline & $\mathrm{N}$ & Mean & Std. Deviation \\
\hline Primary tasks & 68 & 3.68 & .79 \\
\hline Secondary tasks & 69 & 3.80 & .73 \\
\hline Tertiary tasks & 68 & 3.16 & .88 \\
\hline Efficiency-oriented Impacts & 67 & 3.54 & .64 \\
\hline Strategic Impacts & 67 & 3.51 & .61 \\
\hline Effectiveness-oriented impacts & 67 & 3.53 & .68 \\
\hline Work-related Impacts & 67 & 3.19 & .87 \\
\hline Isolation Impacts & 67 & 2.65 & .71 \\
\hline
\end{tabular}

Table 3 shows the descriptive statistics for the study variables. As for CIT use, secondary collaboration tasks were more frequently supported by IT, followed by primary collaboration task and then tertiary collaboration tasks. On the other hand, the highest level of agreement was found for efficiencyoriented impacts of CIT use, followed closely by effectiveness-oriented impacts of CIT use. There was lowest level of agreement between responding organizations on isolation impacts of CIT use.

In order to test for any associations between IT supported collaboration tasks (i.e. CIT use) and their organizational level impacts, a correlation analysis was conducted between the three types of collaborative tasks supported by IT (i.e. primary tasks, secondary tasks, and tertiary tasks) and the five organizational level impacts resulting from the use of IT to support collaboration (i.e. efficiency-oriented, strategic, effectiveness-oriented, work-related, and isolation impacts). Table 4 shows the results of the correlation analysis. Our analysis indicates that all the three IT supported collaboration tasks were found to be significantly and positively correlated with efficiencyoriented impacts, strategic impacts, and effectiveness- 
oriented organizational-level impacts. No significant correlations were found between any of the three IT supported collaborative tasks and work-related and isolation impacts at the organization level. The strongest correlations were found between IT support for primary collaboration tasks and strategic and effectiveness-oriented organizational level impacts. On the other hand, the weakest correlations were found between IT support for tertiary collaboration tasks and efficiency-oriented and effectiveness-oriented collaboration tasks. Our results also indicate that IT support for all the three collaboration tasks does not correlate with negative organizational impacts of CIT use.

Table 4: Correlation analysis

\begin{tabular}{|c|c|c|c|c|c|}
\hline \multicolumn{6}{|c|}{ Organizational Impacts } \\
\hline & Efficiency & Strategic & Effectiveness & Work & Isolation \\
\hline Primary Tasks & $0.31^{* *}$ & $0.51^{* * *}$ & $0.51^{* * *}$ & ns & ns \\
\hline Secondary Tasks & $0.31^{* *}$ & $0.42^{* * *}$ & $0.31^{* *}$ & ns & ns \\
\hline Tertiary Tasks & $0.27^{* *}$ & $0.32^{\star *}$ & $0.28^{* *}$ & ns & ns \\
\hline
\end{tabular}

\section{Discussion and conclusions}

While the majority of the past organizational-level macro studies have explored various CITs that are available on the market, "function-centric" approaches have been rare. Such an approach focuses on collaboration tasks/process and the functionality provided by the technology to support collaboration rather than the specific CIT(s). Since multiple CITs can support multiple tasks through a wide range of functionalities, function-centric approaches may be more useful in understanding CIT use as a broader range of products are deployed to support collaboration.

Our motivation was to explore collaboration tasks that are supported by IT (or CIT use) and the organizational level impacts of CIT use across organizations. The study findings suggest that IT is being used to support many types of collaborative tasks in organizations. From the standpoint of level of use, IT support for collaboration was "always" used for: communication in almost $47 \%$ of our responding firms, information and knowledge sharing in about $24 \%$ of responding firms, and report writing/information pooling in about $18 \%$ of the firms. On the other hand IT support was "always" used for discussion and brainstorming in less than $5 \%$ of the responding organizations. From the standpoint of collaboration, discussion and brainstorming may not always be support by IT and it is possible that these tasks better lend themselves to face-to-face interaction rather than being supported by CIT. We also found that IT was "occasionally" used to support a wide range of collaboration tasks including monitoring progress, planning, time management, and discussion and brainstorming in more than $35 \%$ of the responding organizations. Thus our findings suggest that CIT use (at least occasionally) is gaining popularity in more than a third of our responding organizations.

The findings also suggest that there was far more "agreement" on all the positive impacts of IT supported collaboration work than there was "disagreement". More than $80 \%$ of the firms agreed that IT support for collaboration in their organization had improved their existing business processes and resulted in time savings to accomplish tasks. While a higher percentage of firms disagreed that IT supported collaborative work led to negative impacts, a larger percentage of our respondents agreed that IT support for collaboration led to information overload. This could be a serious concern as CITs become more and more popular in organizational settings.

Overall our findings suggest the use of IT to support collaboration tasks is positively and significantly associated with organizational-level impacts. CIT use can lead to: time saving to accomplish task, bring about quick reaction to changes, lead to faster decision-making, allow more work to be accomplished, and lead organizations to respond more appropriately to changes. From a strategic standpoint, CIT use can lead to improved relationships with external entities such as customers, suppliers, and business partners, resulting in offering the right products and services. Finally, IT supported collaboration can also lead to improved business processes, make organizations "flatter", and can facilitate innovations. These findings have important implications for practice. Given the wide magnitude of organizational level impacts of CITs, managing their assimilation is critical and organizational decision makers must make conscious efforts to encourage 
collaboration. Executives need to establish appropriate control mechanisms to stimulate their proliferation. The impacts of IT supported collaborative work also have important implications for managing organizational change and transformation as CIT use can bring about significant changes in organization structures and business processes. Such transformations may require realigning incentive systems, redistribution of power and authority, and better managing relationships with external entities. Such changes will pose great challenges but at the same time provide opportunities for organizations to become more competitive.

While the majority of the past literature has focused on group-level performance (impact) of CIT use, our study extends existing research by exploring the organizational level impacts of CITs. We must also recognize the limitations of our study. First, a single respondent was used to collect data on IT supported collaborative work and organizational level impacts of CITs. However, the position of our respondents lends some credibility towards this effect. As almost all of them belonged to senior level management positions in Australian organizations, they are likely to be knowledgeable about the study's objectives. Second, a higher response rate would have facilitated a more robust analysis of the validity of our constructs.

Future research needs to further validate our study constructs and explore the organization level impact of CIT use in different regional settings. Australian organizations were selected for this study to initiate our research efforts and we hope to extend the study to other global regions. CITs will also continue to evolve. The emergence of Web 2.0 tools offers even more opportunities to collaborate. Almost all Web 2.0 tools (blogs, wikis, socialware, mash-ups, aggregators, podcasting etc.) foster collaboration by requiring users to participate and interact. While the impact of these emerging social collaborative tools on business organizations is debatable due to their infancy, future research needs to explore whether the new generation of CITs will be able to better support collaborative efforts of end-users within and across organizational boundaries.

\section{References}

[1] Amrit, C. and J. von Hillegersberg, "Detecting coordination problems in collaborative software development environments", Information Systems Management, 25(1), 2008, pp. 57-70.

[2] Applegate, L.M., R. Austin, and F.W. McFarlan, Corporate Information Strategy and Management: Text and Cases, Irwin/McGraw-Hill, Boston, MA, 2006.
[3] Bajwa, D.S., L.F. Lewis, G. Pervan, V.S. Lai, B.E. Munkvold, and G. Schwabe, "Factors related to the global assimilation of collaborative information technologies: an exploratory investigation in five regions", Journal of Management Information Systems, 25(1), 2008, pp. 131-165.

[4] Bayo-Moriones, A and F. Lera-Lopez, "A firmlevel analysis of determinants of ICT adoption in Spain”, Technovation, 27, 2007, pp. 352-366.

[5] Dabbish, L. and R. Kraut, "Awareness displays and social motivation for coordinating communication", Information Systems Research, 19(2), 2008, pp. 221241.

[6] David, G.C., D. Chand, S. Newell, and J. ResendeSantos, "Integrated collaboration across distributed sites: the perils of process and the promise of practice", Journal of Information Technology, 23(1), 2008, pp. 44-54.

[7] Dedrick, J., V. Gurbaxani, and K.L.Kraemer, "Information technology and economic performance: a critical review of the empirical evidence", ACM Computing Surveys, 35(1), 2003, pp. 1-28.

[8] DeLone, W. H. and E.R. McLean, "Information systems success: the quest for the dependent variable", Information Systems Research, 3(1), 1992, pp. 60-95.

[9] DeLone, W.H. and E.R. McLean, "The DeLone and McLean model of information systems success: a ten-year update", Journal of Management Information Systems, 19(4), 2003, pp. 9-30.

[10] Easley, R.F., S. Devaraj, and J.M. Crant, "Relating collaborative technology use to teamwork quality and performance: an empirical analysis", Journal of Management Information Systems, 19(4), 2003, pp. 247-268.

[11] Fedorowicz, J. I. Laso-Ballesteros, and A. PadillaMelendez, "Creativity, innovation, and ecollaboration", International Journal of Ecollaboration, 4(4), October-December 2008, pp. 1-10.

[12] Hair, J.F., R.E. Anderson, W. Black, and R.L. Tatham, Multivariate Data Analysis, $5^{\text {th }}$ edition, Prentice Hall, Upper Saddle River, NJ, 1998.

[13] Hansen, P. and K. Jarvelin, "Collaborative information retrieval in an information-intensive domain", Information Processing \& Management, 41(5), September, 2005, pp. 1101-1119.

[14] Hansen, M.T. and N. Nohria, "How to build collaborative advantage", MIT Sloan Management Review, 46(1), 2004, pp. 22-30. 
[15] Jasperson, J., P.E. Carter, and R.W. Zmud, “A comprehensive conceptualization of post-adoption behaviors associated with information technology enabled work systems", MIS Quarterly, 29(3), 2005, pp. 525-558.

[16] Kahai, S.S., E. Carroll, and R. Jestice, "Team collaboration in virtual worlds", Database for Advances in Information Systems, 38(4), 2007, pp. 6168.

[17] Karsten, H., "Collaboration and collaborative information technologies: a review of the evidence", Database for Advances in Information Systems, 30(2), 1999, pp. 44-66.

[18] Munkvold, B.E and I. Zigurs, "Integration of ecollaboration technologies: research opportunities and challenges", International Journal of e-Collaboration, 1(2), 2005, pp. 1-24.

[19] Nunamaker, J.F. Jr, R.O. Briggs, D.D. Mittleman, D.R. Vogel, and P.A. Balthazard, "Lessons from a dozen years of group support systems research: a discussion of lab and field findings", Journal of Management Information Systems, 13(3), Winter 1996-1997, pp. 163-208.

[20] Nunnally, J.C. Jr, Introduction to Psychological Measurement, McGraw-Hill, New York, 1970.

[21] Pavlou, P.A., A. Dimoka, and T.J. Housel, "Effective use of collaborative IT tools: nature, antecedents, and consequences", Proceedings of the $41^{\text {st }}$ Hawaii International Conference on System Sciences, Hawaii, January 5-9, 2008.
[22] Sarmiento, J.W. and G. Stahl, "Group creativity in interaction: collaborative referencing, remembering and bridging", International Journal of HumanComputer Interaction, 24(5), 2008. pp. 492-504.

[23] Sethi, V. and W. King, "Construct measurement in information systems research: an illustration in strategic systems", Decision Sciences, 22(3), 1991, pp. 455-472.

[24] Siakas, K.V. and E. Siakas, "The need for trust relationships to enable successful virtual team collaboration in software outsourcing", International Journal of Technology Policy and Management, 8(1), 2008, pp. 59-70.

[25] Thomas, D.M., R.P. Bostrom, and M. Gouge, "Making knowledge work in virtual teams", Communications of the ACM, 50(11), 2007, pp. 85-95.

[26] Vandenbosch, B. and M. Ginzberg, "Lotus notes and collaboration: plus ca change.....", Journal of Management Information Systems 3(3), 1996-1997, pp. 65-82.

[27] Weil, N., "The trouble with collaboration tools; everyone wants to collaborate using IT. The problem IT leaders cite: a lack of integration among the tools, and frustrated end-users", CIO, 21(2), 2007, pp. 1. 